\section{Metastasis to the appendix from gastric cancer detected incidentally on colonoscopy}

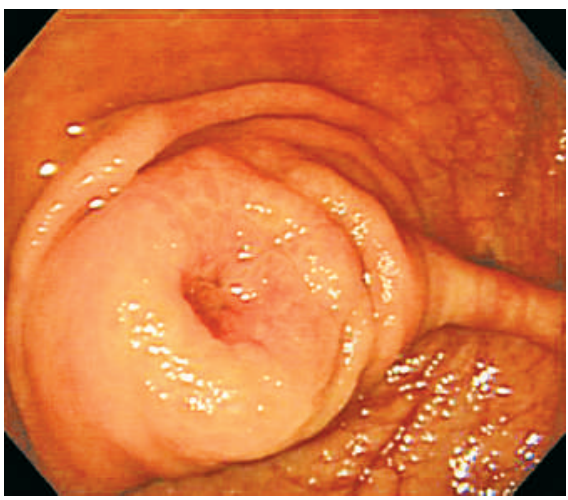

Figure 1 Colonoscopy showed that the appendiceal orifice was generally elevated, as if pushed up by a solid appendiceal mass.

A 59-year-old man complaining of anal pain was referred to our division for investigation. His medical history included a total gastrectomy for advanced poorly differentiated gastric adenocarcinoma, which had penetrated the serosa without invasion of adjacent structures or peritoneal dissemination. Colonoscopy revealed that the appendiceal orifice was generally elevated, as if pushed up by a solid appendiceal mass (Figure $\mathbf{1}$ ). In addition, coarse, reddish mucosa was detected at the appendiceal orifice (Figure 2). Histological examination of the biopsy specimens taken from the mucosa at the appendiceal orifice during colonoscopy revealed poorly differentiated adenocarcinoma, consistent with metastasis from the gastric carcinoma that had been surgically resected 1 year previously. Unfortunately, the patient died 1 year after receiving systemic chemotherapy.

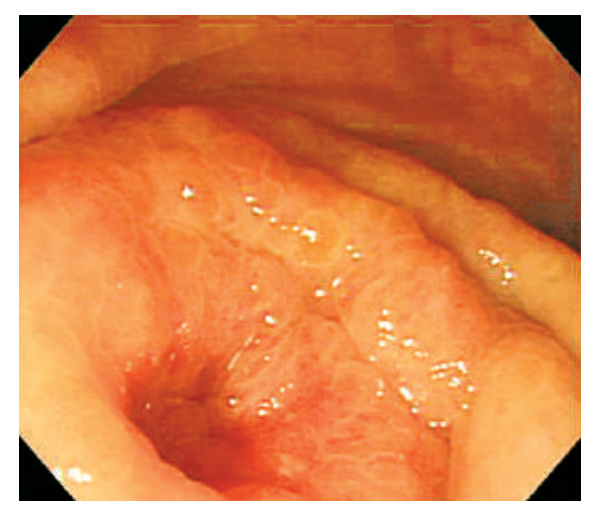

Figure 2 Coarse and reddish mucosa at the appendiceal orifice was detected by colonoscopy.

Metastasis of gastric carcinoma to the appendix is rare, and was first described by Goldfarb and Zuckner in 1951 [1]. None of the reported cases, apart from the present one, was diagnosed correctly before surgery [2]. Clinically, metastatic carcinoma of the appendix is asymptomatic, and patients generally present with symptoms of acute appendicitis, often with perforation. The mechanism of development is considered to be attachment of metastatic tumor cells primarily to the serosa and their infiltration through all of the layers of the appendiceal wall, which becomes enlarged and circumferentially occludes the lumen of the appendix, finally resulting in obstruction and causing secondary inflammation [2]. Unfortunately, patients with metastatic appendiceal tumors usually have a poor prognosis $[1,3]$. Further clinical evaluation of a large number of patients will be necessary in order to clarify whether early detection with endoscopic biopsy and subsequent systemic chemotherapy might improve the survival rate.
Endoscopy_UCTN_Code_CCL_1AD_2AB

K. Fu1, 2, T. Horimatsu' ${ }^{2}$, Y. Sano ${ }^{2}$, A. Katagiri' ${ }^{2}$, Y. Kaji ${ }^{1}$, T. Fujimori ${ }^{3}$

${ }^{1}$ Dept. of Radiology, Dokkyo University School of Medicine, Tochigi, Japan 2 Division of Gastrointestinal Oncology and Digestive Endoscopy, National Cancer Center Hospital East, Chiba, Japan

${ }^{3}$ Dept. of Surgical and Molecular Pathology, Dokkyo University School of Medicine, Tochigi, Japan.

\section{References}

${ }^{1}$ Goldfarb A, Zuckner J. Acute suppurative appendicitis with perforation resulting from metastatic carcinoma: report of a case. Surgery $1951 ; 29: 137-141$

2 Lin CY, Huang JS, Jwo SC, Chen HY. Recurrent gastric adenocarcinoma presenting as acute appendicitis: a case report. Int J Clin Pract Suppl 2005; 59 (Suppl 147): 89-91

${ }^{3}$ Dieter RA. Carcinoma metastatic to the vermiform appendix: report of three cases. Dis Colon Rectum 1970; 13: 336-340

\section{Corresponding author}

KI Fu, M.D., Ph.D.

Dept. of Radiology

Dokkyo University School of Medicine 880 Kitakobaysashi, Mibu, Shimotuga Tochigi 321-0293

Japan

Fax: $\quad+81-282-86-5678$

Email: fukuangi@hotmail.com 